\title{
Autoregressive Cross-Lagged Modeling of The Longitudinal Relationship Between Sub-Health and Cold Hypersensitivity
}

Hee-Jeong Jin ( $\nabla$ hjjin@kiom.re.kr)

Korea Institute of Oriental Medicine https://orcid.org/0000-0002-4373-3410

Sujung Kim

Housing Urban Finance Institute

Siwoo Lee

Korea Institute of Oriental Medicine

\section{Research}

Keywords: ARCLM, cold hypersensitivity, health status

Posted Date: October 6th, 2020

DOI: https://doi.org/10.21203/rs.3.rs-50157/v1

License: (c) (i) This work is licensed under a Creative Commons Attribution 4.0 International License.

Read Full License 


\section{Abstract}

Background: Sub-health refers to abnormal symptoms that occur in the body, but with no specific disease, and a high probability of future diseases. Meanwhile, cold hypersensitivity is one of the most basic diagnosis methods used in Oriental medicine for explaining a patient's health status, and various studies have supported its association with health status. The present study analyzed differences in cold hypersensitivity regarding health status and examined changes in health status and cold hypersensitivity over time.

Methods: The health status and cold hypersensitivity of 255 adults from Daejeon in South Korea were examined. To assess the longitudinal relationship between health status and cold hypersensitivity, data at baseline, 6-month follow-up, and 12-month follow-up were used for analysis using an autoregressive cross-lagged model (ARCLM).

Results: ARCLM analysis showed that the path coefficients of each autoregressive coefficient (baseline to 6-month follow-up, 6-month follow-up to 12-month follow-up) of health status and cold hypersensitivity moving from measurement time point $t$ to $t+1$ were significant in all time intervals. The same was true for health status patterns. Thus, these findings suggested that health status and cold hypersensitivity were not transient symptoms but persisted over time. The findings also showed that health status had a significant cross-lagged effect on cold hypersensitivity.

Conclusions: Health status and cold hypersensitivity were found to be stable and associated with each other over time. Therefore, consistent care is necessary to maintain health status and treat cold hypersensitivity. The longitudinal data used in this study to examine the causal relationship between health status and cold hypersensitivity demonstrate the diagnostic value of cold hypersensitivity and the need for health status-based interventions.

\section{Background}

Advances in science, technology, and medicine have extended the average life expectancy of humans. The global population is rapidly aging. The WHO (World Health Organization) has estimated $(1,2)$ the percentage of older people in the global population will increase from 11\% in 2000 to $22 \%$ in 2050 . In Korea, according to a report by the Korea National Statistical Office, the percentage of people aged 65 years and older in the population has continued to increase from $3.1 \%$ in 1970 to $12.2 \%$ in 2013, and this number is expected to grow to $24.3 \%$ by 2030 and $37.4 \%$ by 2050 , thereby creating a super-aged society (3). As average life expectancy increases, interest in health care is also growing, with the greatest importance placed on disease prevention. Recently, there has been an increasing trend in complaints of being in a state of "sub-health." Sub-health refers to the state of having abnormal symptoms without any specific disease, with the risk of developing future diseases. In modern medicine, this is described as "a state between health and disease," and involves psychological, physical, and medical components believed to increase the risk of illness $(4,5)$. 
Each person has a different sensitivity to cold even when they share the same environment (6). In Oriental medicine, cold sensitivity which is called as cold pattern identification, represents one of the eight components of the principle of hypersensitivity identification or syndrome differentiation (7). It is used as a major tool for identifying disease characteristics, patient condition, and appropriate treatment methods. The cold pattern (CP) is characterized by a preference for warm temperature, intolerance of cold, hypothermia, paleness, diarrhea, peripheral chills, and spasms (8). Especially, cold hypersensitivity in the hands and feet (CHHF) is a condition in which affected individuals feel excessively cold at low temperatures because of spastic peripheral vasoconstriction. Cold hypersensitivity can be prominent in certain diseases, for example in hand injuries or Flammer syndrome (FS) $(9,10)$. In cold hypersensitivity, the skin temperature of the extremities is negatively correlated with that of the abdomen $(11,12)$. In a recent study, it was seen that CHHF, independent of one's body mass, could induce increased levels of circulating adiponectin, and was associated with metabolic syndrome (MS) (12). Cold hypersensitivity may lower one's quality of life (13). In the case of elderly patients with upper extremity trauma, they experience various problems after an acute hand injury. Many of these problems diminish during the first year after the injury (14). However, in a ten year follow up study, pain and cold intolerance were reported as long-standing problems in patients after hand trauma (15), and cold intolerance was found to be the variable that had the most impact on quality of life (16). However, studies on differences in cold indices according to quality of life and health status are still lacking.

The present study divided healthy adults into three groups based on their health status and analyzed differences in cold indices between these groups, in addition to changes in their health status and cold hypersensitivity over time. The findings in the present study were expected to serve as basic data on the diagnostic value of cold hypersensitivity for a subject's health status

\section{Methods}

\section{Participants}

This longitudinal study included faculty members of Daejeon University located in Daejeon, Korea, who were examined three times at 6-month intervals between July 2015 and December 2016. Symptom questionnaires as well as cold-hypersensitivity data were derived from the Korean Medicine Data Center (KDC) of the Korea Institute of Oriental Medicine. The study included 255 participants from 30 to 59 years of age, and written consent was obtained from all participants prior to the study. The inclusion criteria were as follows: 1 ) men and women aged $30-59$ years, 2 ) individuals who provided informed consent. However, individuals diagnosed with cancer (malignant tumor) were excluded. This study was approved by the Institutional Review Board of Daejeon University (1040647-201603-HR-001-03 (2016)), and conducted according to the principles expressed in the Declaration of Helsinki.

Out of all the possible participants, 26 were excluded based on exclusion criteria, resulting in 379 participants. Of these, 114 participants were lost to follow-up at visit 2 (6 months) and 10 were lost at 
visit 3 follow-up (12 months). A total of 255 participants who completed all three questionnaires were included in the final analyses (Fig. 1).

\section{Measurements}

\section{Health status}

For determining health status, the abnormal symptoms-based Mibyeong Index (MBI) developed by the Korea Institute of Oriental Medicine (KIOM) was used (S1 Appendix) $(17,18)$, which has also been utilized in previous research (19). The questionnaire consisted of symptoms frequently experienced by the general population in Korea (fatigue $70.7 \%$, pain $30.8 \%$, sleep disturbance $16.7 \%$, dyspepsia $18.3 \%$, depression $17.3 \%$, anger $18.7 \%$, and anxiety $12.8 \%)(18,20)$.

The severity of each symptom, the duration, and recovery were measured on a 7-point scale and the questionnaire comprised a total of 21 questions. The summarized score by symptom type ranges between 3 and 21 points, and the total MBI of 7 symptoms ranges between 21 and 147 points. Lower scores on the MBI indicate a healthy state and higher scores indicate more severe sub-health (20). For the health status assessment, the MBI was divided into quartiles with the 1st quartile classified as the Healthy group, the 2nd and 3rd quartile classified as the Sub-Health 1" (SH1) group, and the 4th quartile classified as the Sub-Health 2" (SH2) group (19).

\section{Cold hypersensitivity}

The cold hypersensitivity questionnaire used in the present study (S2 Appendix) was tested for reliability and validity in earlier studies $(21,22)$. The questionnaire comprised eight questions on cold hypersensitivity. Responses to each question were evaluated on a 5-point scale with 1 point indicating "not at all"; 2 points "a little bit"; 3 points "average"; 4 points "somewhat"; and 5 points indicating "very much so." The cut-off value of cold hypersensitivity was 21.5 with an agreement of $87.1 \%$. The diagnosis was performed by two Korean medicine doctors (KMDs) with more than 5 years of clinical experience, and the subjects included 93 adults (22).

\section{Health-Related Quality of Life}

HRQOL was collected at baseline using the 12-Item Short-Form Health Survey (SF-12), which is a reduced version of the SF-36 questionnaire $(23,24)$. The SF-12 is a health-related questionnaire with established validity and reliability. It is divided into the physical component summary (PCS) and the mental component summary (MCS), and consists of eight domains. Scores range from 0 to 100 points, with a higher score indicating better health.

\section{Statistical analyses}

To examine the general characteristics of the participants, the present analysis used chi-square tests on categorical data and one-way ANOVA on continuous data. In addition, Spearman's correlation analysis was performed to examine the correlation between health status and cold hypersensitivity, while logistic 
regression analysis was performed to examine whether health status has an impact on cold hypersensitivity.

To examine the directionality of the longitudinal causal relationship between health status and cold hypersensitivity, data at baseline, 6-month follow-up, and 12-month follow-up were analyzed using the ARCLM (autoregressive cross-lagged model) method. The key features of ARCLM are that the model can explain the time point $t+1$ variable by the time point $t$ variable, and that the model can be expanded to a multivariate model to simultaneously test for any mutual effect, that is, a cross-lagged effect, between the two variables $(25,26)$.

In the present study, associations between time points $t$ and $t+1$ for health status and cold hypersensitivity were estimated by an autoregressive model. Moreover, for the cross-lagged effects, correlations between health status at time point $t$ and cold hypersensitivity at time point $t+1$, as well as between cold hypersensitivity at time point $t$ and health status at time point $t+1$, were estimated. The present study used the SPSS statistical package, version 22.0 (IBM, Chicago, IL, USA) for data processing and basic statistical analysis, while the ARCLM was implemented using AMOS 21.0 (IBM, Chicago, IL, USA).

\section{Results}

\section{Descriptive statistics}

The participants' sex distribution in the present study was 122 males (47.8\%) and 133 females (52.2\%). With respect to the sex distribution of each group, the healthy, $\mathrm{SH} 1$, and $\mathrm{SH} 2$ groups contained 52 (57.8\%), 38 (47.5\%), and 32 (37.6\%) males, and thus, 38 (42.2\%), 42 (52.5\%), and $53(62.4 \%)$ females, respectively.

Mean age $(p<0.001)$ in the healthy, $\mathrm{SH} 1$, and $\mathrm{SH} 2$ groups was $41.0 \pm 8.9,44.9 \pm 8.6$, and $48.0 \pm 10.3$ years, respectively. The values for MCS $(p<0.001)$, indicating mental quality of life, and PCS, indicating physical quality of life, showed significant differences according to health status (healthy group: MCS: $53.0 \pm 6.2$, PCS: $51.4 \pm 5.2$; SH1 group: MCS: $48.0 \pm 7.9$, PCS: $48.3 \pm 7.1$; SH2 group: MCS: $42.2 \pm$ 8.1, PCS: $45.9 \pm 8.0)$.

Among the members of the healthy, SH1, and SH2 groups, 64 (71.1\%), 52 (65.0\%), and 68 (80.0\%) participants responded that they were current drinkers $(p=0.096)$, while $9(10.0 \%), 11(13.8 \%)$, and 15 $(17.6 \%)$ participants responded that they were current smokers $(p=0.34)$, respectively. In all three groups, 2.50-4.99 million KRW (Korean Won) was the most common response for household income level, and university (university or higher) was the most common response for education level (Table 1). 
Table 1

Baseline characteristics of the study participants

\begin{tabular}{|c|c|c|c|c|c|}
\hline \multirow[t]{2}{*}{ Variable } & \multirow[t]{2}{*}{ Categories } & \multirow{2}{*}{$\begin{array}{l}\text { Healthy } \\
N=90\end{array}$} & \multirow{2}{*}{$\begin{array}{l}\text { SH1 } \\
N=80\end{array}$} & \multirow{2}{*}{$\begin{array}{l}\mathrm{SH} 2 \\
\mathrm{~N}=85\end{array}$} & \multirow[t]{2}{*}{ p-value } \\
\hline & & & & & \\
\hline \multirow[t]{2}{*}{ Sex } & Male & $52(57.8)$ & $38(47.5)$ & $32(37.6)$ & \multirow[t]{2}{*}{0.029} \\
\hline & Female & $38(42.2)$ & $42(52.5)$ & $53(62.4)$ & \\
\hline Age & - & $41.0 \pm 8.9$ & $44.9 \pm 8.6$ & $48.0 \pm 10.3$ & $<0.001$ \\
\hline \multirow[t]{2}{*}{ BMI } & Male & $25.3 \pm 3.1$ & $25.2 \pm 3.0$ & $25.4 \pm 3.1$ & 0.974 \\
\hline & Female & $23.5 \pm 3.1$ & $22.3 \pm 3.1$ & $22.9 \pm 3.8$ & 0.31 \\
\hline MCS & - & $53.0 \pm 6.2$ & $48.0 \pm 7.9$ & $42.2 \pm 8.1$ & $<0.001$ \\
\hline PCS & - & $51.4 \pm 5.2$ & $48.3 \pm 7.1$ & $45.9 \pm 8.0$ & $<0.001$ \\
\hline \multirow[t]{2}{*}{ Drinking } & Yes & 64(71.1) & $52(65.0)$ & $68(80.0)$ & \multirow[t]{2}{*}{0.096} \\
\hline & NO & $26(28.9)$ & $28(35.0)$ & $17(20.0)$ & \\
\hline \multirow[t]{2}{*}{ Smoking } & Yes & $9(10.0)$ & 11(13.8) & $15(17.6)$ & \multirow[t]{2}{*}{0.34} \\
\hline & NO & $81(90.0)$ & $69(86.2)$ & $70(82.4)$ & \\
\hline \multirow[t]{3}{*}{ Household income $^{a}$} & $\leq 2.49$ & $23(31.1)$ & $15(22.5)$ & $26(32.9)$ & \multirow[t]{3}{*}{0.438} \\
\hline & $2.50 \sim 4.99$ & $47(35.6)$ & $43(53.7)$ & $43(49.4)$ & \\
\hline & $\geq 5.00$ & $20(33.3)$ & $22(23.8)$ & 16(17.7) & \\
\hline \multirow[t]{3}{*}{ Education } & $\leq$ Middle school & $7(7.8)$ & $0(0.0)$ & $0(0.0)$ & \multirow[t]{3}{*}{0.006} \\
\hline & High school & 10(11.1) & $7(8.8)$ & $12(14.1)$ & \\
\hline & $\geq$ University & 73(81.1) & 73(91.2) & 73(85.9) & \\
\hline \multirow[t]{2}{*}{ Marital status } & With spouse & 78(86.7) & $67(83.8)$ & $62(72.9)$ & \multirow[t]{2}{*}{0.052} \\
\hline & Without spouse & $12(13.3)$ & 13(16.2) & $23(27.1)$ & \\
\hline
\end{tabular}

\section{Association between health status and cold hypersensitivity}

Prior to analyzing the ARCLM, Spearman's correlation analysis was performed to investigate the correlation between health status and cold hypersensitivity (Tables 2 ). The analysis results show that, overall, health status as well as cold hypersensitivity measured during each visit showed significant levels of positive correlation. These results indicated that it would be appropriate to test the causal relationship between health status and cold hypersensitivity. 
Table 2

Correlation between health status and cold hypersensitivity

\begin{tabular}{|c|c|c|c|c|c|c|}
\hline & BHS & $6 \mathrm{MHS}$ & $12 \mathrm{MHS}$ & $\mathrm{BCH}$ & $6 \mathrm{MCH}$ & $12 \mathrm{MCH}$ \\
\hline BHS & 1.000 & & & & & \\
\hline $6 \mathrm{MHS}$ & $0.603^{\star *}$ & 1.000 & & & & \\
\hline $12 \mathrm{MHS}$ & $0.472^{\star \star}$ & $0.571^{* *}$ & 1.000 & & & \\
\hline $\mathrm{BCH}$ & $0.179 \star \star$ & $0.208^{* *}$ & 0.067 & 1.000 & & \\
\hline $6 \mathrm{MCH}$ & $0.297^{\star \star}$ & $0.390 * *$ & $0.507^{\star *}$ & $0.233^{* *}$ & 1.000 & \\
\hline $12 \mathrm{MCH}$ & $0.327^{\star *}$ & $0.490^{* \star}$ & $0.194 * \star$ & $0.280^{\star *}$ & $0.381^{\star \star}$ & 1.000 \\
\hline
\end{tabular}

\section{Cold hypersensitivity according to health status}

To identify the relationship between health status and cold hypersensitivity, the present study used logistic regression at baseline, adjusted for sex, age, and BMI (Table 3).

The $\mathrm{SH} 1$ and $\mathrm{SH} 2$ groups had a probability of having cold hypersensitivity that was $\mathrm{OR}=2.141(\mathrm{p}=$ $0.024)$ and $O R=2.618(p=0.006)$ times higher than that for the Healthy group, respectively.

Table 3

Cold hypersensitivity according to health status at baseline

\begin{tabular}{|llccc|}
\hline Health status & p-value & OR & 95\% C.I. & \\
\hline ref. Health & 0.014 & & & \\
\hline SH 1 & 0.024 & 2.141 & 1.105 & 4.149 \\
SH 2 & 0.006 & 2.618 & 1.310 & 5.231 \\
\hline Health, healthy group; SH1, sub-health 1 group; SH2, sub-health 2 group; OR: Odds Ratio. \\
\hline
\end{tabular}

\section{ARCL modeling results}

To investigate the longitudinal causal relationship between health status and cold hypersensitivity, the present study used the ARCLM method. The longitudinal relationship between health status and cold hypersensitivity was tested by fitting a three-wave autoregressive cross-lagged model (Fig. 2) at three time points: baseline, 6-month follow-up, and 12-month follow-up.

The goodness of fit of the ARCLM was assessed using a goodness of fit index (GFl; > 0.90 acceptable, > 0.95 excellent), a normed fit index (NFI; > 0.90 acceptable, > 0.95 excellent), the Tucker-Lewis index (TLI; > 0.90 acceptable, $>0.95$ excellent), a comparative fit index (CFl; $>0.90$ acceptable, > 0.95 excellent), and 
standardized root-mean-square residuals (SRMR; $<0.08$ acceptable $<0.05$ excellent) $(27-29)$. The indices for cold hypersensitivity were all favorable, with $\mathrm{GFI}=0.979, \mathrm{NFI}=0.962, \mathrm{TLI}=0.888, \mathrm{CFI}=0.97$, and SRMR $=0.039$, indicating that the model had an excellent fit. The analysis showed that the path coefficients of each autoregressive coefficient of health status (baseline $\rightarrow 6$-month follow-up: $\beta=0.584$, $p<0.001$; 6-month follow-up $\rightarrow$ 12-month follow-up: $\beta=0.623, p<0.001)$ and cold hypersensitivity (baseline $\rightarrow 6$-month follow-up: $\beta=0.229, p<0.001 ; 6$-month follow-up $\rightarrow 12$-month follow-up: $\beta=0.25, p$ $<0.001$ ) going from measurement time point $t$ to $t+1$ were significant at all time intervals (Table 4;

Fig. 2). With respect to the cross-lagged coefficients between health status and cold hypersensitivity, BHS $\rightarrow 6 \mathrm{MCH}(\beta=0.286, p<0.001), 6 \mathrm{MHS} \rightarrow 12 \mathrm{MCH}(\beta=0.268, \mathrm{p}<0.001)$, and $\mathrm{BCH} \rightarrow 6 \mathrm{MHS}(\beta=0.103, \mathrm{p}=$ 0.042 ) were all significant.

Table 4

Cross-lagged autoregressive modeling between health status and cold hypersensitivity

\begin{tabular}{|c|c|c|c|c|c|c|c|}
\hline \multirow[b]{2}{*}{$\begin{array}{l}\text { Health status and cold } \\
\text { hypersensitivity }\end{array}$} & \multicolumn{3}{|c|}{ Pathways } & \multirow{2}{*}{$\begin{array}{l}\text { Estimate } \\
0.584\end{array}$} & \multirow{2}{*}{$\begin{array}{l}\text { S.E. } \\
0.051\end{array}$} & \multirow{2}{*}{$\begin{array}{l}\text { C.R. } \\
11.569\end{array}$} & \multirow{2}{*}{$\begin{array}{l}\begin{array}{l}\text { p- } \\
\text { value }\end{array} \\
< \\
0.001\end{array}$} \\
\hline & BHS & $\rightarrow$ & $6 \mathrm{MHS}$ & & & & \\
\hline & $\mathrm{BCH}$ & $\rightarrow$ & $6 \mathrm{MCH}$ & 0.229 & 0.059 & 3.919 & $\begin{array}{l}< \\
0.001\end{array}$ \\
\hline & BHS & $\rightarrow$ & $6 \mathrm{MCH}$ & 0.286 & 0.035 & 4.88 & $\begin{array}{l}< \\
0.001\end{array}$ \\
\hline & $\mathrm{BCH}$ & $\rightarrow$ & $6 \mathrm{MHS}$ & 0.103 & 0.086 & 2.038 & 0.042 \\
\hline & $6 \mathrm{MHS}$ & $\rightarrow$ & $12 \mathrm{MHS}$ & 0.623 & 0.045 & 10.616 & $\begin{array}{l}< \\
0.001\end{array}$ \\
\hline & $6 \mathrm{MCH}$ & $\rightarrow$ & $12 \mathrm{MCH}$ & 0.25 & 0.064 & 3.889 & $\dot{0} 001$ \\
\hline & $6 \mathrm{MHS}$ & $\rightarrow$ & $12 \mathrm{MCH}$ & 0.268 & 0.038 & 4.159 & $\begin{array}{l}< \\
0.001\end{array}$ \\
\hline & $6 \mathrm{MCH}$ & $\rightarrow$ & $12 \mathrm{MHS}$ & -0.11 & 0.077 & -1.88 & 0.06 \\
\hline
\end{tabular}

\section{Discussion}

The present study divided adults, 30-59 years of age, into three groups based on their health status, to assess the relationship between health status and cold hypersensitivity over time, and to examine differences in indices between the groups.

With respect to sex differences in health status, we found more males in the healthy group (male: $57.8 \%$ > female: $42.2 \%$ ), while the percentage of females increased with condition severity, such as in the $\mathrm{SH} 2$ 
group (male: $37.6 \%<$ female: $62.4 \%)(p=0.029)$. Mean age was highest in the $\mathrm{SH} 2$ group $(48.0 \pm 10.3)$, followed by the SH1 $(44.9 \pm 8.6)$ and the healthy $(41.0 \pm 8.9)$ group $(p<0.001)$. These findings are consistent with an earlier study which reported that males take physical illness or injury less seriously and are less sensitive than females, and with an existing study that reported that an increase in age leads to physical and mental illnesses (30-32).

We found a significant positive correlation between health status and cold hypersensitivity that were followed up in two 6-month intervals, which indicates that having poor health results in having cold hypersensitivity. To identify the relationship between health status and cold hypersensitivity, the present study used logistic regression at baseline, adjusted for sex, age, and BMI. The BMI was included as it has been shown to have high correlation with cold hypersensitivity in previous studies on BMI and constitution type $(33,34)$. The results were similar in that when the participants' health status was poorer, their probability of having cold hypersensitivity was higher. These findings are similar to those of another study reporting that a group of diseased patients had cold hypersensitivity on the exterior (35), and are consistent with poorer health status being associated with higher cold hypersensitivity scores (36).

The ARCLM results on health status and cold hypersensitivity showed that the stability coefficients of health status, expressed as autoregressive coefficients, appeared static over time. When health status was measured at three time points, the results showed that the health status at one time point had a significant influence on the health status at a subsequent time point. In other words, health status is not a transient state, but a state that persists over time. This is consistent with results from longitudinal panel surveys in middle-aged people, which have indicated that baseline health status does not show significant change over time $(37,38)$. Cold hypersensitivity also appeared to be stable over time, and the cold hypersensitivity at a specific time point had a significant influence on the cold hypersensitivity later. In other words, a person who has cold hypersensitivity does not have transient symptoms of cold hypersensitivity, but symptoms that persist over time. Our investigation of the cross-lagged effects of health status and cold hypersensitivity over time showed that health status had a stable and significant effect on cold hypersensitivity over time, while cold hypersensitivity had a partially significant effect on health status. These results indicate that poorer health status increases the probability of cold hypersensitivity. On the other hand, cold hypersensitivity did not appear to have a major influence on health status. These findings are similar to earlier results showing that the degree of phase angle (PA), which assesses health status by taking into account the condition of cell membranes, was lower in a cold-hypersensitivity group than in a non-cold-hypersensitivity group (38-40).

Several limitations of this study must be considered when interpreting the results. First, the sample population was relatively small, especially for a longitudinal analysis. However, all applicable longitudinal data were included without any exclusion criteria. The findings in the present study should be validated with a bigger sample population and a longer follow-up period. Second, the participants in the present study were restricted to faculty members from the Daejeon region in South Korea, which might limit the generalizability of the findings. The response rate to the questionnaire survey, however, was high and the survey included diverse age groups. Third, because health status and cold hypersensitivity were 
investigated using a questionnaire survey, there might have been limitations regarding the correct classification of the respondents due to implicit biases associated with self-reporting. However, many studies use self-diagnostic tools to measure various symptoms and diseases (20). Fourth, the measurement process was not comprehensive in scope and did not include social and environmental aspects. The findings are therefore limited to cold hypersensitivity, focusing on health status as an implicating factor. However, the relationship between the two non-recursive variables, health status and cold hypersensitivity, was empirically tested via three-wave bivariate autoregressive cross-lagged modeling.

The results of the present study identify health status as a variable that has a significant influence on cold hypersensitivity suggesting that future studies on the prevention and treatment of symptoms caused by cold hypersensitivity should focus on the patient's general health status, along with other major variables.

\section{Conclusion}

Health status and cold hypersensitivity appear to be stable over time, indicating that health status and cold hypersensitivity are not transient symptoms but long lasting ones. The influence of health status on cold hypersensitivity in our study sample was stable and significant over time, which suggests that people with severe sub-health conditions may be more susceptible to this hypersensitivity. Future longitudinal studies will be needed to observe changes in symptoms over a longer period of time, as well as investigations into the mutual associations between various factors, such as demographic and disease factors.

\section{List Of Abbreviations}

ARCLM

Autoregressive cross-lagged model; HRQOL:Health-Related Quality of Life; MS:Metabolic syndrome; PSC:Physical summary component; MSC:Mental summary component; MBI:Mibyeong Index; CHHF:Cold hypersensitivity in the hands and feet; $\mathrm{SH} n$ :sub-health $n$ group; BHS:baseline health status; $n \mathrm{MHS}: n$ month follow-up health status; $\mathrm{BCH}$ :baseline cold hypersensitivity; $n \mathrm{MCH}$ : $n$-month follow-up cold hypersensitivity; OR:Odds Ratio; S.E.:Standard error of unstandardized coefficients; C.R.:Critical ratio; C.I.:Confidence Interval; S.E.; Standard error of unstandardized coefficients; $\beta$, Standardized beta coefficients

\section{Declarations}

\section{Ethics approval and consent to participate}

Written consent was obtained from all participants prior to the study. This study was approved by the Institutional Review Board of Daejeon University (1040647-201603-HR-001-03 (2016)), and conducted 
according to the principles expressed in the Declaration of Helsinki.

\section{Consent for publication}

Not Applicable.

\section{Availability of data and materials}

Please contact author for data requests.

\section{Competing interests}

The authors have declared that no competing interests exist.

\section{Funding}

This study was supported by the "Development of Korean Medicine Original Technology for Preventive Treatment based on Integrative Big Data" grant from the Korea Institution of Oriental Medicine (no. KSN2021120).

\section{Authors' contributions}

SK analyzed and interpreted the data, and drafted the manuscript. SL participated in the design of the study and the preparation of the manuscript. HJ conceived the study and participated in its design and coordination. All authors read and approved the final manuscript.

\section{Acknowledgments}

Not Applicable

\section{References}

1. Facts about ageing: World Health Organization; [Available from: http://www.who.int/ageing/about/facts/en/. Accessed 03/07/2020.

2. Organization WH. World report on ageing and health: World Health Organization; 2015.

3. Korea's Population: Commissioner of Statistics Korea (KOSTAT); [Available from: http://kostat.go.kr/portal/eng. Accessed 03/07/2020.

4. Dunstan R, Sparkes D, Macdonald M, De Jonge XJ, Dascombe B, Gottfries J, et al. Diverse characteristics of the urinary excretion of amino acids in humans and the use of amino acid supplementation to reduce fatigue and sub-health in adults. Nutrition journal. 2017;16(1):19.

5. Li G, Xie F, Yan S, Hu X, Jin B, Wang J, et al. Subhealth: definition, criteria for diagnosis and potential prevalence in the central region of China. BMC Public Health. 2013;13(1):446. 
6. Hur Y-M, Chae J-H, Chung KW, Kim JJ, Jeong H-U, Kim JW, et al. Feeling of cold hands and feet is a highly heritable phenotype. Twin Research and Human Genetics. 2012;15(2):166-9.

7. Organization WH. WHO international standard terminologies on traditional medicine in the western pacific region. 2007.

8. Hur Y-M, Yu H, Jin H-J, Lee S. Heritability of cold and heat patterns: A twin study. Twin Research and Human Genetics. 2018;21(3):227-32.

9. Carlsson I, Cederlund R, Höglund P, Lundborg G, Rosén B. Hand injuries and cold sensitivity: reliability and validity of cold sensitivity questionnaires. Disability and rehabilitation. 2008;30(25):1920-8.

10. Barthelmes J, Nägele MP, Ludovici V, Ruschitzka F, Sudano I, Flammer AJ. Endothelial dysfunction in cardiovascular disease and Flammer syndrome-similarities and differences. EPMA Journal. 2017;8(2):99-109.

11. Yasui T, Uemura H, Irahara M, Arai M, Kojimahara N, Okabe R, et al. Differences in sensitivity to cold in japanese men and postmenopausal women aged $\geq 50$ years. Gender medicine. 2007;4(4):359-66.

12. Park AY, Cha S. Effects of cold sensitivity in the extremities on circulating adiponectin levels and metabolic syndrome in women. BMC complementary and alternative medicine. 2017;17(1):150.

13. Brandow AM, Stucky CL, Hillery CA, Hoffmann RG, Panepinto JA. Patients with sickle cell disease have increased sensitivity to cold and heat. American journal of hematology. 2013;88(1):37-43.

14. Gustafsson M, Ahlström G. Problems experienced during the first year of an acute traumatic hand injury-a prospective study. Journal of clinical nursing. 2004;13(8):986-95.

15. Gustafsson M, Hagberg L, Holmefur M. Ten years follow-up of health and disability in people with acute traumatic hand injury: pain and cold sensitivity are long-standing problems. Journal of Hand Surgery (European Volume). 2011;36(7):590-8.

16. Reitan I, Dahlin LB, Rosberg H-E. Patient-reported quality of life and hand disability in elderly patients after a traumatic hand injury-a retrospective study. Health and quality of life outcomes. 2019;17(1):148.

17. Lee J, Kim S-H, Lee Y, Song S, Kim Y, Lee S. The concept of Mibyeong (sub-health) in Korea: A Delphi study. European Journal of Integrative Medicine. 2013;5(6):514-8.

18. Lee E, Lee Y, Park K, Yoo J, Lee S. A study on recognition of Mibyeong and its prevalence in Korean public: national survey. Journal of Society of Preventive Korean Medicine. 2015;19(3):1-10.

19. Lee Y, Baek Y, Park K, Jin H-J, Lee S. Development and validation of an instrument to measure the health status of healthy but unsatisfied people: Mibyeong index. Journal of Society of Preventive Korean Medicine. 2016;20(3):45-53.

20. Mun S, Park K, Baek Y, Lee S, Yoo J-h. Interrelationships among common symptoms in the elderly and their effects on health-related quality of life: a cross-sectional study in rural Korea. Health and quality of life outcomes. 2016;14(1):146.

21. Bae K-H, Yoon Y, Yeo M, Kim H-S, Lee Y, Lee S. Development on the Questionnaire of Cold-Heat Pattern Identification Based on Usual Symptoms for Health Promotion-Focused on Agreement Study. 
Journal of Society of Preventive Korean Medicine. 2016;20(2):17-26.

22. Yeo M, Park K, Bae K, Jang E, Lee Y. Development on the Questionnaire of Cold-Heat Pattern Identification Based on Usual Symptoms for Health Promotion-Focused on Reliability Study. Journal of Physiology \& Pathology in Korean Medicine. 2016;30(2):116-23.

23. Ware Jr JE, Kosinski M, Keller SD. A 12-Item Short-Form Health Survey: construction of scales and preliminary tests of reliability and validity. Medical care. 1996;34(3):220-33.

24. Vilagut G, Valderas J, Ferrer M, Garin O, López-García E, Alonso J. Interpretation of SF-36 and SF-12 questionnaires in Spain: physical and mental components. Medicina clinica. 2008;130(19):726-35.

25. Hertzog C, Nesselroade JR. Beyond autoregressive models: Some implications of the trait-state distinction for the structural modeling of developmental change. Child Development. 1987:93-109.

26. Sher KJ, Wood MD, Wood PK, Raskin G. Alcohol outcome expectancies and alcohol use: A latent variable cross-lagged panel study. Journal of abnormal psychology. 1996;105(4):561.

27. Ho S-Y, Rohan KJ, Parent J, Tager FA, McKinley PS. A longitudinal study of depression, fatigue, and sleep disturbances as a symptom cluster in women with breast cancer. Journal of pain and symptom management. 2015;49(4):707-15.

28. Hu Lt, Bentler PM. Cutoff criteria for fit indexes in covariance structure analysis: Conventional criteria versus new alternatives. Structural equation modeling: a multidisciplinary journal. 1999;6(1):1-55.

29. Lix LM, Wu X, Hopman W, Mayo N, Sajobi TT, Liu J, et al. Differential item functioning in the SF-36 physical functioning and mental health sub-scales: a population-based investigation in the Canadian multicentre osteoporosis study. PloS one. 2016;11(3).

30. Cho S-J, Oh D-H, Park Y-C, Nam J-H, Kim S-H. The clinical association between the prevalence of insomnia and physical and psychiatric disorders in two areas of Gyeonggi Province of South Korea. Journal of Korean Neuropsychiatric Association. 2016;55(1):41-50.

31. Hilderink PH, Collard R, Rosmalen JG, Oude Voshaar RC. How does ageing affect the impact of medically unexplained symptoms and medically explained symptoms on health-related quality of life? International journal of geriatric psychiatry. 2015;30(7):737-43.

32. Rustøen T, Wahl AK, Hanestad BR, Lerdal A, Paul S, Miaskowski C. Age and the experience of chronic pain: differences in health and quality of life among younger, middle-aged, and older adults. The Clinical journal of pain. 2005;21(6):513-23.

33. Xain HM LW, Li Z. The correlation analysis of children with different constitution types and BMI. Medical Innovation of China. 2016;2016(24):108-10.

34. Mozaffarieh M, Gasio PF, Schötzau A, Orgül S, Flammer J, Kräuchi K. Thermal discomfort with cold extremities in relation to age, gender, and body mass index in a random sample of a Swiss urban population. Population Health Metrics. 2010;8(1):17.

35. Kim J-E, Lee S-G, Ryu H-S, Park K-M. Differences of cold-heat patterns between healthy and disease group. Journal of physiology \& pathology in Korean Medicine. 2006;20(1):224-8. 
36. Kim S, Lee S, Lee Y. A Study on the Difference of Cold-heat Patterns between Health and Mibyeong Group. Journal of Society of Preventive Korean Medicine. 2017;21(1):49-56.

37. Yu C. A longitudinal analysis on subjective health status and life satisfaction in the middle-old aged. Korean Journal of Gerontological Social Welfare. 2015;68:331-56.

38. Guida B, Laccetti R, Gerardi C, Trio R, Perrino NR, Strazzullo P, et al. Bioelectrical impedance analysis and age-related differences of body composition in the elderly. Nutrition, Metabolism and Cardiovascular Diseases. 2007;17(3):175-80.

39. Jha V, Jairam A, Sharma M, Sakhuja V, Piccoli A, Parthasarathy S. Body composition analysis with bioelectric impedance in adult Indians with ESRD: comparison with healthy population. Kidney international. 2006;69(9):1649-53.

40. Siddiqui NI, Khan SA, Shoeb M, Bose S. Anthropometric predictors of bio-impedance analysis (BIA) phase angle in healthy adults. Journal of clinical and diagnostic research: JCDR. 2016;10(6):CC01.

\section{Figures}

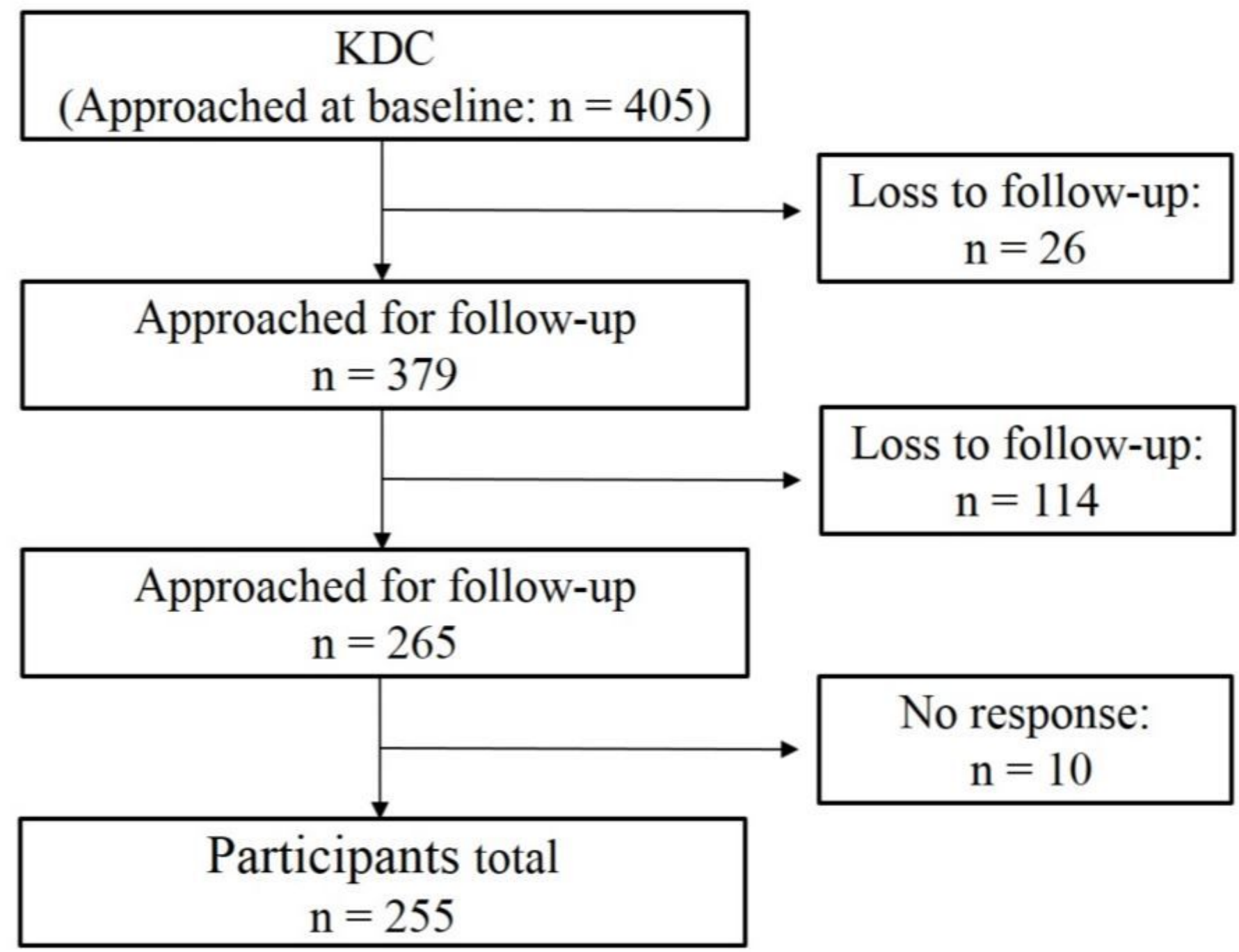


Figure 1

Flow chart of participant selection and loss to follow-up.

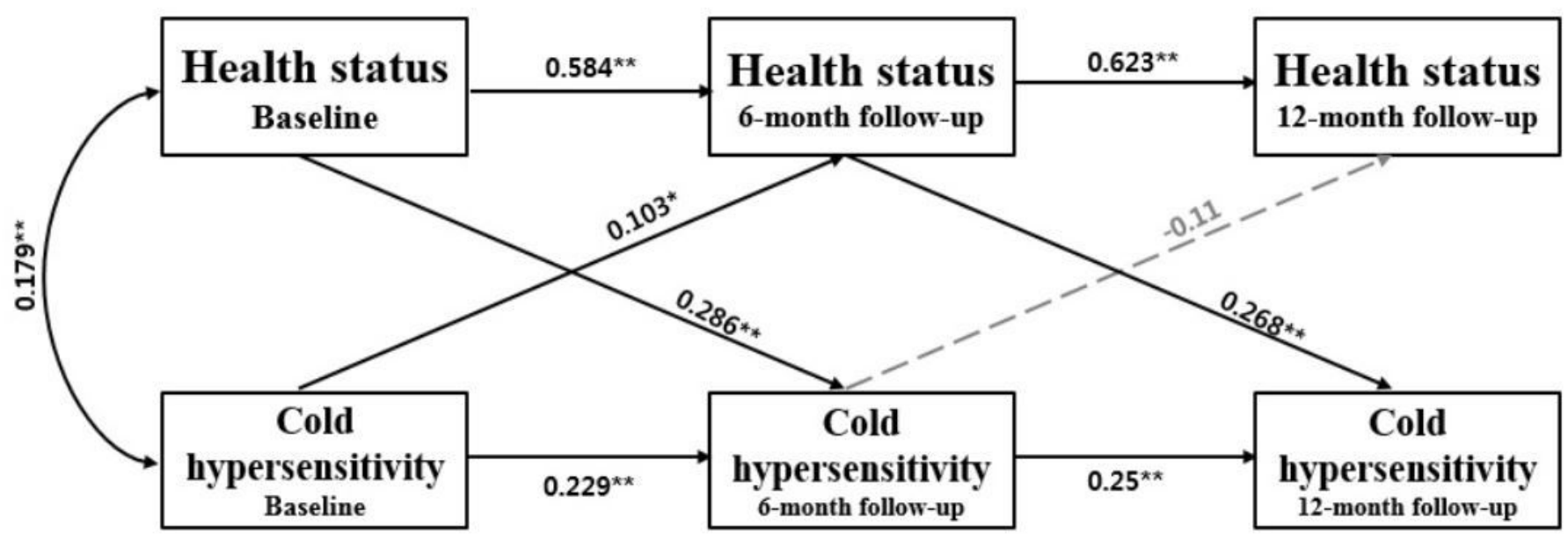

Figure 2

Cross-lagged autoregressive modeling between health status and cold hypersensitivity. ${ }^{*} p<0.05$, $\star \star p<0.001$.

\section{Supplementary Files}

This is a list of supplementary files associated with this preprint. Click to download.

- Appendix.docx 